\title{
Applicability of a Single 5 Color Cytoplasmic Markers Tube as Primary Panel in Routine Immunophenotyping of Acute Leukemia
}

Rahul Kumar Sharma, Vandana Puri, Deepti Mutreja, Sunil Kumar, Pratibha Chauhan, Abhishek Purohit, Prabhu Manivannan, Sudha Sazawal, Pravas Mishra and Renu Saxena*

Department of Hematology, All India Institute of Medical Sciences, New Delhi, India

*Corresponding author: Renu Saxena, Department of Hematology, All India Institute of Medical Sciences, New Delhi, India, Tel: 011-2659-4670; E-mail: renusaxena@outlook.com

Received date: Aug 17, 2015, Accepted date: Sep 24, 2015, Publication date: Sep 28, 2015

Copyright: @ 2015 Sharma RK, et al. This is an open-access article distributed under the terms of the Creative Commons Attribution License, which permits unrestricted use, distribution, and reproduction in any medium, provided the original author and source are credited.

\begin{abstract}
Background: Immunophenotyping in acute leukemia is a routine practice for lineage assignment. Conventionally a primary panel with surface markers are applied first followed by cytoplasmic markers as secondary panel in the diagnosis of acute leukemia.We in this present study aim to assess the relevance of a single 5 color "CD45, MPO, CD79a, CD3, Tdt" cytoplasmic markers combination to be utilized as primary panel.
\end{abstract}

Methods: Sensitivity and specificity of different subset of positive negative combination of these markers were retrospectively analyzed in the 458 acute leukemia cases.

Results: MPO or cCD3 positivity alongwith cCD79a negativity was $100 \%$ specific diagnosis for AML and T-ALL respectively. Furthermore, cCD79a positivity along with MPO and CCD3 negativity was $97.2 \%$ specific for B-ALL diagnosis. MPO and CCD79a dual positivity was found $100 \%$ sensitive and $92.6 \%$ specific for MPAL (B-My) diagnosis. MPO and CCD3 dual positivity was 100\% sensitive and specific for MPAL (T-My) diagnosis.

Conclusion: We found a good correlation of this single tube diagnosis when compared with standard morphology, cytochemistry, and flow cytometry based diagnosis. We hope our this cytoplasmic panel may help to design a precise extended immunophenotypic panel for acute leukemia diagnosis and may also be a cost effective approach in resource constrained developing countries.

Keywords: Acute leukemia; Flow cytometry; Immunophenotyping; Cytoplasmic antigens

\section{Introduction}

Immunophenotypic diagnosis of acute leukemia is performed based on a panel of lineage associated markers including cell surface as well as cytoplasmic antigens [1-3]. The standard approach is to apply first, primary panel of markers with cell-surface antigens then after secondary panel of markers with cytoplasmic antigens [3-5]. However, multiple reports have shown that cytoplasmic markers are the earliest identifiable marker of a particular cell development and they come on cell surface later $[3,6,7]$. Prior Information of cytoplasmic markers expression in the leukemic blasts may direct to apply a well systematic and extended secondary panel for accurate acute leukemia diagnosis. Such type of reports are very little available and nil in Indian Territory. We in this present study tried to investigate the diagnostic relevance of a single 5 color tube containing the markers MPO, cCD79a, cCD3, TdT (along with CD45-gating marker) as primary panel for acute leukemia immunophenotyping by comparing its sensitivity and specificity with standard morphology, cytochemistry and flow cytometry based diagnosis.

\section{Materials and Methods}

This study was conducted in the Department of Hematology, All india Institute of Medical Sciences, New Delhi, India during the period of January 2011 to December 2012. All the 458 cases of acute leukemia i.e ALL (B-ALL and T-ALL), AML and MPAL consecutively enrolled in this two year period were included in the study. All the cases were enrolled only after getting their written consent. Diagnosis of acute leukemia was based on morphological examination of bone marrow aspirate smears, including cytochemistry along with full panel of flow cytometric immunophenotyping. Almost of the samples received in our laboratory were bone marrow aspirate which were processed using a standard stain-lyse-wash method. All the samples were processed within 24 hour of collection. The monoclonal antibodies used in full panel were: CD45 (PerCP-Cy5.5), CD13 (PE), CD33 (APC), CD10 (PE-Cy7), CD19 (FITC), CD7 (FITC), CD117 (APC), CD34 (PE-Cy7), HLA-DR (PE), MPO (FITC), c CD79a (PE), c CD3 (PE-Cy7), Tdt (APC), CD64 (FITC) and CD11c (APC). The cytoplasmic tube contained CD45 as gating marker and MPO, CD3, CD79a, Tdt. All of these monoclonal antibodies were purchased from $\mathrm{BD}$ Biosciences (San Jose, CA, USA). Initial incubation of monoclonal antibodies for 15-30 min at room temperature in the dark followed by lysis and washing of debris/unlysed RBCs were performed and samples were acquired using a BD-FACS Canto (6 color, 2 LASER) system. For the cytoplasmic tube an additional step by incubating it with permeablizing solution (BD Biosciences) was carried out to render the easy passage of intracellular markers in cytoplasm of cells. An unstained control tube without adding any monoclonal antibody was prepared to set the quadrant for autofluorescence exclusion. All analyses and interpretation were carried out using the FACS-Diva software (BD Biosciences). 
Citation: Sharma RK, Puri V, Mutreja D, Kumar S, Chauhan P, et al. (2015) Applicability of a Single 5 Color Cytoplasmic Markers Tube as Primary Panel in Routine Immunophenotyping of Acute Leukemia. J Blood Disord Transfus 6: 309. doi:10.4172/2155-9864.1000309

Page 2 of 5

\section{Results}

Out of the total 458 acute leukemia patients recruited, 222 were diagnosed as AML, 176 as B-ALL, 44 as T-ALL and 16 as MPAL by standard morphology, cytochemistry and immunphenotyping based criteria. We retrospectively analyzed the applicability of various intracellular combinations of immunophenotypic markers for independent diagnosis compared with that of final combinatorial diagnosis. The 13 different intracellular immune phenotypic subset were made based on positive and negative status of each marker (Table 1).

Since we did not see positive or negative status of Tdt playing any crucial role in the diagnosis, we further shortlisted this Table by ignoring the Tdt status (Table 2). These 6 different subsets of cytoplasmic markers positive negative status as found in Table 2 were comprehensively examined separately for their diagnostic utility in AML, B-ALL, T-ALL, MPAL (B-My), MPAL (T-My) diagnosis.

\begin{tabular}{|c|c|c|c|c|c|c|}
\hline \multirow[t]{2}{*}{ S.N } & \multirow{2}{*}{$\begin{array}{l}\text { Intracellular Immunophenotypic } \\
\text { expression subset }\end{array}$} & \multirow{2}{*}{$\begin{array}{l}\text { AML } \\
(n=222)\end{array}$} & \multirow{2}{*}{$\begin{array}{l}\text { B-ALL } \\
\text { (n=176) }\end{array}$} & \multirow{2}{*}{$\begin{array}{l}\text { T-ALL } \\
(n=44)\end{array}$} & \multicolumn{2}{|l|}{ MPAL } \\
\hline & & & & & $\begin{array}{l}\text { B-My } \\
(n=14)\end{array}$ & $\begin{array}{l}\text { T-My } \\
(n=2)\end{array}$ \\
\hline 1 & $\mathrm{MPO}^{+} \mathrm{cCD}^{-19 a^{-}} \mathrm{cCD}^{-} \mathrm{Tdt}^{-}$ & $126(56.7)$ & $0(0)$ & $0(0)$ & $0(0)$ & $0(0)$ \\
\hline 2 & $\mathrm{MPO}^{+} \mathrm{cCD}^{-} 9 \mathrm{a}^{-} \mathrm{cCD}^{-} \mathrm{Tdt}^{+}$ & $52(23.4)$ & $0(0)$ & $0(0)$ & $0(0)$ & $0(0)$ \\
\hline 3 & $\mathrm{MPO}^{+} \mathrm{cCD}^{2} \mathrm{a}^{+} \mathrm{cCD}^{-} \mathrm{Tdt}^{+}$ & $9(4.0)$ & $0(0)$ & $0(0)$ & $9(64.2)$ & $0(0)$ \\
\hline 4 & $\mathrm{MPO}^{+} \mathrm{cCD} \mathrm{Ca}^{+} \mathrm{cCD}^{-} \mathrm{Tdt}^{-}$ & $9(4.0)$ & $0(0)$ & $0(0)$ & $5(35.7)$ & $0(0)$ \\
\hline 5 & $\mathrm{MPO}^{-} \mathrm{cCD} 79^{+} \mathrm{cCD}^{-} \mathrm{Tdt}^{-}$ & $8(3.6)$ & $21(11.9)$ & $0(0)$ & $0(0)$ & $0(0)$ \\
\hline 6 & 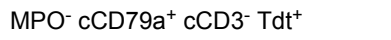 & $0(0)$ & $153(86.9)$ & $0(0)$ & $0(0)$ & $0(0)$ \\
\hline 7 & 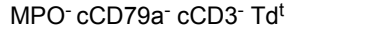 & $12(5.4)$ & $0(0)$ & $0(0)$ & $0(0)$ & $0(0)$ \\
\hline 8 & $\mathrm{MPO}^{-} \mathrm{cCD}^{-} 9^{-} \mathrm{cCD}^{-} \mathrm{Tdt}^{+}$ & $6(2.7)$ & $02(1.1)$ & $0(0)$ & $0(0)$ & $0(0)$ \\
\hline 9 & MPO- cCD79a- cCD3 ${ }^{+} \mathrm{Tdt}^{+}$ & $0(0)$ & $0(0)$ & $11(25)$ & $0(0)$ & $0(0)$ \\
\hline 10 & $\mathrm{MPO}^{-} \mathrm{cCD}{ }^{+}{ }^{+} \mathrm{cCD}^{+} \mathrm{Tdt}^{+}$ & $0(0)$ & $0(0)$ & $17(38.6)$ & $0(0)$ & $0(0)$ \\
\hline 11 & $\mathrm{MPO}^{-} \mathrm{cCD} \mathrm{Ca}^{+} \mathrm{cCD}^{+} \mathrm{Tdt}^{-}$ & $0(0)$ & $0(0)$ & $8(18.1)$ & $0(0)$ & $0(0)$ \\
\hline 12 & $\mathrm{MPO}^{-} \mathrm{cCD}^{-} 9^{-} \mathrm{cCD}^{+}{ }^{-} \mathrm{Tdt}^{-}$ & $0(0)$ & $0(0)$ & $8(18.1)$ & $0(0)$ & $0(0)$ \\
\hline 13 & $\mathrm{MPO}^{+} \mathrm{cCD} \mathrm{Ca}^{-} \mathrm{cCD}^{+} \mathrm{Tdt}^{+}$ & $0(0)$ & $0(0)$ & $0(0)$ & $0(0)$ & $2(100)$ \\
\hline
\end{tabular}

Table 1: Different subset of tubes with variable positive negative expression of cytoplasmic markers.

\begin{tabular}{|c|c|c|c|c|c|c|}
\hline \multirow[t]{2}{*}{ S.N. } & \multirow{2}{*}{$\begin{array}{l}\text { Intracellular Immunophenotypic } \\
\text { expression subset }\end{array}$} & \multirow{2}{*}{$\begin{array}{l}\text { AML } \\
(n=222)\end{array}$} & \multirow{2}{*}{$\begin{array}{l}B-A L L \\
n=176)\end{array}$} & \multirow{2}{*}{$\begin{array}{l}\text { T-ALL } \\
(n=44)\end{array}$} & \multicolumn{2}{|l|}{ MPAL } \\
\hline & & & & & $\begin{array}{l}\text { B-My } \\
(n=14)\end{array}$ & $\begin{array}{l}\text { T-My } \\
(n=2)\end{array}$ \\
\hline 1 & $\mathrm{MPO}^{-} \mathrm{cCD}^{+} \mathrm{cCD}^{-} 9 \mathrm{a}^{+/-} \mathrm{Tdt}^{+/-}$ & $0(0)$ & $0(0)$ & $44(100)$ & $0(0)$ & $0(0)$ \\
\hline 2 & MPO- cCD79a ${ }^{+}$cCD3 $\mathrm{Tdt}^{+/-}$ & $8(3.6)$ & $174(98.8)$ & $0(0)$ & $0(0)$ & $0(0)$ \\
\hline 3 & $\mathrm{MPO}^{+} \mathrm{cCD}^{-} 9 \mathrm{a}^{-} \mathrm{cCD}^{-} \mathrm{Tdt}^{+/-}$ & $178(80.1)$ & $0(0)$ & $0(0)$ & $0(0)$ & $0(0)$ \\
\hline 4 & $\mathrm{MPO}^{+} \mathrm{cCD}^{-} 9 \mathrm{a}^{+} \mathrm{cCD}^{-} \mathrm{Tdt}^{+/-}$ & $18(8.1)$ & $0(0)$ & $0(0)$ & $14(100)$ & $0(0)$ \\
\hline 5 & MPO- cCD79a- cCD3- $\mathrm{Tdt}^{+/-}$ & $18(8.1)$ & $2(1.1)$ & $0(0)$ & $0(0)$ & $0(0)$ \\
\hline 6 & $\mathrm{MPO}^{+} \mathrm{cCD}^{2} 9 \mathrm{a}^{-} \mathrm{cCD}^{+} \mathrm{Tdt}^{+/-}$ & $0(0)$ & $0(0)$ & $0(0)$ & $0(0)$ & $2(100)$ \\
\hline
\end{tabular}

Table 2: Different subset of cytoplasmic tubes with variable positive negative expression after ignoring Tdt status. Subset-1 had $100 \%$ sensitivity and $100 \%$ specificity for T-ALL diagnosis; Subset-2 had $98.8 \%$ sensitivity and $97.2 \%$ specificity for B-ALL diagnosis; Subset-3 had $80.1 \%$ sensitivity and $100 \%$ specificity for AML diagnosis; Subset-5 had 8.1\% sensitivity and 99.1 specificity for AML diagnosis; Subset-3\&5 in combination had $88.3 \%$ sensitivity and $99.1 \%$ specificity for AML diagnosis; Subset-4 had $100 \%$ sensitivity and $92.6 \%$ specificity for MPAL (BMy) diagnosis; Subset- 6 had 100\% sensitivity and 100\% specificity for MPAL (T-My) diagnosis. 
Page 3 of 5

\section{AML subgroup}

Out of total 222 AML patients diagnosed, 196 (88.3\%) were positive for MPO, rest $26(11.71 \%)$ were negative for MPO. AML with MPO positivity exhibited 4 different expression subset of intracellular markers. $\mathrm{MPO}^{+} \mathrm{cCD} 7 \mathrm{a}^{-} \mathrm{cCD}^{-} \mathrm{Tdt}^{-}$expression subset was detected in $126(56.7 \%)$ cases which was nil in all other cases. $\mathrm{MPO}^{+} \mathrm{CCD}^{2} \mathrm{a}^{-}$

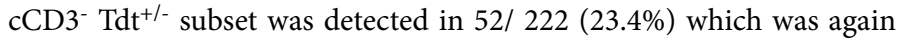
nil for all other cases. $\mathrm{MPO}^{+} \mathrm{cCD} \mathrm{a}^{+} \mathrm{cCD}^{-} \mathrm{Tdt}^{+}$subset was detected in $9(4 \%)$ cases which was nil in B-ALL, T-ALL, MPAL (T/My) but was detected in $9(64.2 \%)$ cases of MPAL (B/My). $\mathrm{MPO}^{+} \mathrm{cCD}^{2} \mathrm{a}^{+} \mathrm{CCD}^{-}$ Tdt- subset was found in 9 (4\%) AML cases which was nil in B-ALL, TALL, MPAL (T/My) but detected in 5 (35.7\%) cases of MPAL (B/My) also.

MPO negative subgroup of AML exhibited 3 different expression subset of intracellular markers. $\mathrm{MPO}^{-} \mathrm{cCD}^{-{ }^{+}} \mathrm{cCD}^{-} \mathrm{Tdt}^{-}$subset was detected in $8(3.6 \%)$ cases of AML which was also expressed by 21 (11.9\%) B-ALL but absent in T-ALL or MPAL. MPO- $^{-} \mathrm{cDD}^{-} \mathrm{a}^{-} \mathrm{cCD}^{-}$ Tdt subset was detected in 12 (5.4\%) AML which was nil in all other cases. $\mathrm{MPO}^{-} \mathrm{cCD}^{-}{ }^{-} \mathrm{cCD}^{-} \mathrm{Tdt}^{+}$was detected in 6 (2.7\%) AML cases, which was also expressed by $2(1.1 \%)$ B-ALL and nil in T-ALL and MPAL.

Total 67 (30.45\%) of AML patients were found to have Tdt coexpression and $26(11.81 \%)$ with cCD79a co-expression. $100 \%$ of the AML were negative for $\mathrm{CCD} 3$ co-expression. Tdt expression was found non-contributory for myeloid lineage determination. 18 (8.10\%) AML cases had MPO positivity along with cCD79a co-expression which was also seen in all $14(100 \%)$ cases of MPAL (B-My) thus making their discrimination difficult. cCD79a co-expression was detected in 8 (3.63\%) AML patients having MPO negativity which overlapped with $21(11.9 \%)$ cases of B-ALL.

By ignoring the positive/negative status of Tdt, 4 different subsets of intracellular expression could be achieved. $\mathrm{MPO}^{+} \mathrm{cCD} \mathrm{Ca}^{-} \mathrm{cCD}^{-} \mathrm{Tdt}$ +/- expression subset was $80.1 \%$ sensitive and $100 \%$ specific for AML diagnosis. $\mathrm{MPO}^{-} \mathrm{cCD}^{-} \mathrm{a}^{-} \mathrm{cCD}^{-} \mathrm{Tdt}^{+/-}$subset was $8.1 \%$ sensitive and 99.1\% specific. $\mathrm{MPO}^{-} \mathrm{cCD}^{2} 9 \mathrm{a}^{+} \mathrm{cCD} 3 \mathrm{Tdt}^{+/-}$subset was $3.6 \%$ sensitive and $26.3 \%$ specific. $\mathrm{MPO}^{+} \mathrm{cCD}^{\mathrm{C}} \mathrm{aa}^{+} \mathrm{cCD}^{-} \mathrm{Tdt}^{+/-}$subset was $8.1 \%$ sensitive and $85.38 \%$ specific. Combination of these 4 subsets had $100 \%$ sensitivity but only $25.42 \%$ specificity for AML diagnosis. However, an intracellular combination of first two subsets only i.e $\mathrm{MPO}^{+} \mathrm{cCD} \mathrm{aa}^{-} \mathrm{cCD}^{-} \mathrm{Tdt}^{+/-}$and $\mathrm{MPO}^{-} \mathrm{cCD}^{-} \mathrm{a}^{-} \mathrm{cCD}^{-} \mathrm{Tdt}^{+/-}$ together had $88.3 \%$ sensitivity and $99.15 \%$ specificity for AML and cases diagnosed by this were found concordant with final combinatorial morphology, cytochemistry and immunophenotyping based diagnosis.

\section{B-ALL subgroup}

In the total $176 \mathrm{~B}$-ALL patients, 3 different intracellular subset of immunophenotypic expression could be detected. $\mathrm{MPO}^{-} \mathrm{cCD}^{+} 9^{+}$ cCD3 $^{-} \mathrm{Tdt}^{-}$expression was detected in 21 (11.9\%) of B-ALL, 8 (3.6\%) of AML and nil in T-ALL and MPAL subgroup. $\mathrm{MPO}^{-} \mathrm{cCD}^{-\mathrm{CD}^{+}}{ }^{+} \mathrm{CD} 3^{-}$ $\mathrm{Tdt}^{+}$expression was detected in 153 (86.9\%) of B-ALL which was nil for all other cases. $\mathrm{MPO}^{-} \mathrm{cCD}^{-}{ }^{-} \mathrm{cCD}^{-} \mathrm{Tdt}^{+}$expression was found in 2 (1.1\%) B-ALL, 6 (2.7\%) AML and nil in T-ALL and MPAL. These 3 intracellular expression subset in combination had $100 \%$ sensitivity and $89.36 \%$ specificity to detect B-ALL. cCD79a expression was found highly sensitive as $98.9 \%$ B-ALL cases expressed it. Tdt expression had $88.06 \%$ sensitivity in B-ALL. All of the B-ALL cases were negative for MPO as well as cCD3. By ignoring the positive/negative status of Tdt, a single intracellular combination with $\mathrm{MPO}^{-} \mathrm{cCD} \mathrm{Ca}^{+} \mathrm{cCD}^{\mathrm{Tdt}} \mathrm{Td}^{+/-}$was found to have $98.8 \%$ sensitivity and $97.2 \%$ specificity for the diagnosis of B-ALL.

\section{T-ALL subgroup}

4 different intracellular immunophenotypic expression subsets were seen in the total $44 \mathrm{~T}$-ALL patients. $\mathrm{MPO}^{-} \mathrm{cCD}^{-} \mathrm{a}^{-} \mathrm{cCD}^{+} \mathrm{Tdt}^{+}$ expression was detected in 11 (25\%) T-ALL which was nil in all other cases. $\mathrm{MPO}^{-} \mathrm{cCD}^{+} 9^{+} \mathrm{cCD}^{+} \mathrm{Tdt}^{+}$was detected in 17 (38.6\%) T-ALL

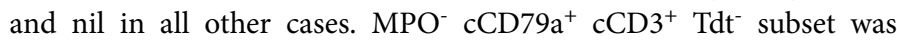
detected in 8 (18.1\%) T-ALL and nil in all other cases. MPO- cCD79- $^{-}$ $\mathrm{cCD}^{+}{ }^{+} \mathrm{Tdt}^{-}$expression was found in $8(18.1 \%)$ T-ALL which was again nil in all other cases. Combination of these 4 different subsets had $100 \%$ sensitivity and $100 \%$ specificity for diagnosis of T-ALL. cCD3 expression was detected in $100 \%$ cases of T-ALL and its co-expression could not be seen anywhere else. Tdt was found co-expressed in $63.6 \%$ and CCD79a in $56.8 \%$ cases of T-ALL. By ignoring positive/negative status of $\mathrm{CCD} 79 \mathrm{a}$ and $\mathrm{Tdt}$, a distinctive immunophenotypic subset of

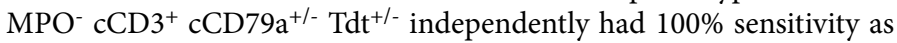
well as $100 \%$ specificity for T-ALL diagnosis.

\section{MPAL subgroup}

Out of total 458 acute leukemia cases recruited, 16 (3.5\%) cases were diagnosed as Mixed phenotypic acute leukemia (MPAL) and 14 $(87.5 \%)$ among them were MPAL (B-My) and $2(12.5 \%)$ were MPAL (T-My). In the 14 MPAL (B-My) subgroup, two immunophenotypic subsets were found. $\mathrm{MPO}^{+} \mathrm{cCD} 79 \mathrm{a}^{+} \mathrm{cCD}^{-} \mathrm{Tdt}^{+}$expression subset was detected in 9 (64.2\%) MPAL (B-My), and also in 9 (4\%) AML, but nil in all other cases. MPO+ $\mathrm{cCD} 79 \mathrm{a}+\mathrm{cCD} 3-\mathrm{Tdt}$ expression subset was detected in 5 (35.7\%) MPAL (B-My) cases, and also in 9 (4\%) B-ALL, but nil in all other cases. We found expression of Tdt in $9(64.2 \%)$ and cCD79a in 100\% MPAL (B-My) cases. By ignoring the positive/ negative status of Tdt, a single intracellular subset with $\mathrm{MPO}^{+} \mathrm{CCD} 79 \mathrm{a}$ $+\mathrm{cCD}^{-} \mathrm{Tdt}^{+/-}$immunophenotype had $100 \%$ sensitivity and $92.6 \%$ specificity for MPAL (B-My) diagnosis. In the total 2 MPAL (T-My) patient subgroup, both cases had the identical intracellular expression subset i.e $\mathrm{MPO}^{+} \mathrm{cCD} 7 \mathrm{a}^{-} \mathrm{cCD}^{+} \mathrm{Tdt}^{+}$which had $100 \%$ sensitivity as well as $100 \%$ specificity for MPAL (T-My) diagnosis.

\section{Discussion}

Flow cytometry based immunophenotpying plays a crucial role in accurate classification and diagnosis of acute leukemia and has become a routine tool in hematologic laboratories worldwide [1-7]. An extensive panel of cell surface as well as intracellular CD markers are required for precise lineage assignment and subtyping of acute leukemia. Intracellular immunophenotypic markers are reported to be expressed at earliest phase of disease and holds a remarkable specificity for myeloid and lymphoid lineage determination in acute leukemia blasts $[3,6,7]$. Conventional practice is to use cell-surface markers as primary panel followed by cytoplasmic markers in second line to establish the diagnosis of acute leukemia [3-5]. Increasing number of the laboratories now a days are rapidly transforming from 3-4 to 5-6 color flow cytometry. We in this present study tried to attempt the applicability of a single 5 color cytoplasmic marker tube of the combination MPO, cCD79a, cCD3, TdT as primary panel and correlated the diagnosis made by this tube with that of final diagnosis made based on standard combinatorial morphology, cytochemistry and flow cytometry. By having multiple positive-negative combinations of all the cytoplasmic markers as demonstrated in Table 
2, we found its higher degree of correlation with the final diagnosis. MPO or CCD3 positivity alongwith cCD79a negativity heralded $100 \%$ specific diagnosis of AML and T-ALL respectively. Furthermore, cCD79a positivity along with MPO and CCD3 negativity was $97.2 \%$ specific for B-ALL diagnosis. a single intracellular combination with $\mathrm{MPO}^{-} \mathrm{cCD}^{-} \mathrm{a}^{+} \mathrm{cCD} 3 \mathrm{Tdt}^{+/-}$was found to have $98.8 \%$ sensitivity and $97.2 \%$ specificity for the diagnosis of B-ALL. A single intracellular combination of $\mathrm{MPO}^{-} \mathrm{cCD}^{+} \mathrm{cCD} \mathrm{Ca}^{+/-} \mathrm{Tdt}^{+/-}$independently had $100 \%$ sensitivity as well as $100 \%$ specificity for T-ALL diagnosis. A single tube of $\mathrm{MPO}^{+} \mathrm{cCD} \mathrm{Ca} \mathrm{a}^{-} \mathrm{cCD}^{-} \mathrm{Tdt}^{+/-}$expression subset was $80.1 \%$ sensitive and $100 \%$ specific for AML diagnosis. MPO negative AML were little a bit tough to assign, although negativity of all the cytoplasmic markers was observed $99.1 \%$ specific for AML diagnosis. MPO and cCD79a dual positivity was found $100 \%$ sensitive and $92.6 \%$ specific for MPAL (B-My) diagnosis and a single tube of $\mathrm{MPO}^{+}$ $\mathrm{cCD} \mathrm{a}^{+} \mathrm{cCD}^{-} \mathrm{Tdt}^{+/-}$immunophenotype had $100 \%$ sensitivity and 92.6\% specificity for MPAL (B-My) diagnosis. Similarly, MPO and cCD3 dual positivity was $100 \%$ sensitive and specific for MPAL (T-My) diagnosis as a single tube with $\mathrm{MPO}^{+} \mathrm{cCD} \mathrm{a}^{-} \mathrm{cCD}^{+} \mathrm{Tdt}^{+}$ combination had $100 \%$ sensitivity as well as $100 \%$ specificity for MPAL (T-My) diagnosis.

Since at the very immature stage of development the antigens are expressed in cytoplasm first then after they manifest to the cell surface a primary panel for lineage affiliation and then secondary panel to determine degree of cell differentiation is standard approach for acute leukemia immunophenotyping [3-7]. Studies have indicated intracellular expression of MPO, cCD3, cCD79a and $\mathrm{cCD} 22$ are the earliest expressing myeloid, B and T cell markers [8-13]. Some studies have also reported that CD19 is not the first differentiation antigen in human B-cell development $[14,15]$. B-cells were shown to be grown from a progenitor pool with $\mathrm{CD} 19^{-} \mathrm{CD} 34^{+} \mathrm{CD} 10^{+}$cells which support the concept of immaturity markers gaining at the earliest stage [10]. Immunophenotypic features in early $\left(\mathrm{CD} 34^{+} \mathrm{TDT}^{+}\right)$lymphoid cells have demonstrated that many of these precursors which lack CD19, they already express CCD79a in combination with CD10 [16]. These types of reports led us to comprehensively evaluate the diagnostic relevance of these cytoplasmic markers as primary panel in acute leukemia immunophenotyping. Our results are in support of these reports as our $98.9 \%$ B-ALL cases expressed CD79a and 100\% T-ALL cases expressed cCD3 (Table 3). MPO expression in AML was seen in $100 \%$ of AML after excluding MO, M5 and M7 subtype which are known to characteristically lack it (Table 3 ). Similar finding were also reported by Drach et al. [17]. Tdt expression as immaturity marker was rather confounding and did not help much in lineage assignment. Our results strongly support that lineage specificity in acute leukemia at a greater extent can be determined by cytoplasmic antigens of the cells and their application as primary panel in routine immunophenotyping laboratories may be an attractive approach of implementation, although pertaining the necessity of full cell surface marker combination for the confirmation and proper classification. Our 5 color cytoplasmic markers based combination may also help a lot as cost-effective method of leukemia diagnosis in the resourceconstrained developing countries.

\begin{tabular}{|l|l|l|l|l|l|}
\hline Diagnosis & Total no. of cases & MPO & cCD79a & cCD3 & Tdt \\
\hline AML & 222 & $196(88.3 \%)$ & $26(11.71 \%)$ & $0(0 \%)$ & $67(30.45 \%)$ \\
\hline B-ALL & 176 & $0(0 \%)$ & $174(98.9 \%)$ & $0(0 \%)$ & $155(88.1 \%)$ \\
\hline T-ALL & 44 & $0(0 \%)$ & $25(56.81 \%)$ & $44(100 \%)$ & $28(63.64 \%)$ \\
\hline MPAL (B-My) & 14 & $14(100 \%)$ & $14(100 \%)$ & $0(0 \%)$ & $9(64.2 \%)$ \\
\hline MPAL (T-My) & 2 & $2(100 \%)$ & $0(0 \%)$ & $2(100 \%)$ & $2(100 \%)$ \\
\hline
\end{tabular}

Table 3: Frequency of different cytoplasmic markers in their particular lineages.

\section{Conclusion}

In nutshell, we in the present study evaluated the applicability of a single 5 color cytoplasmic tube based methodology to use it as primary panel in routine acute leukemia immunophenotyping. We found a good correlation of this single tube based diagnosis with final diagnosis made on the basis of standard morphology, cytochemistry and flow cytometry assessment. We assume this single tube based methodology will be very helpful in designing needful secondary extended panel protocol for precise diagnosis of acute leukemia and could also be particularly useful as cost-effective approach in the resource constrained countries.

\section{References}

1. Pui CH, Behm FG, Crist WM (1993) Clinical and biologic relevance of immunologic marker studies in childhood acute lymphoblastic leukemia. Blood 82: 343-362.

2. Borowitz MJ (1990) Immunologic markers in childhood acute lymphoblastic leukemia. Hematol Oncol Clin North Am 4: 743-765.
3. Knapp W, Strobl H, Majdic O (1994) Flow cytometric analysis of cellsurface and intracellular antigens in leukemia diagnosis. Cytometry 18: 187-198.

4. Slaper-Cortenbach ICM, Admiraal LG, Kerr JM, van Leeuwen EF, Von dem Borne AE, et al. (1988) Flow cytometric detection of terminal deoxynucleotidyl transferase and other intracellular antigens in combination with membrane antigens in acute lymphatic leukemias. Blood 72: 1639-1644.

5. Pombo de Oliveira MS, Matutes E, Rani S, Morilla R, Catovsky D (1988) Early expression of MCS2 (CD13) in the cytoplasm of blast cells from acute myeloid leukaemia. Acta Haematol 80: 61-64.

6. Mirro J Jr, Zipf TF, Pui CH, Kitchingman G, Williams D, et al. (1985) Acute mixed lineage leukemia: clinicopathologic correlations and prognostic significance. Blood 66: 1115-1123.

7. Janossy G, Coustan-Smith E, Campana D (1989) The reliability of cytoplasmic CD3 and CD22 antigen expression in the immunodiagnosis of acute leukemia: a study of 500 cases. Leukemia 3: 170-181.

8. Van der Schoot CE, Daams GM. Pinkster J, Vet R, Von dem Borne AE (1990) Monoclonal antibodies against myeloperoxidase are valuable immunological reagents for the diagnosis of acute myeloid leukemia. $\mathrm{Br} \mathrm{J}$ Haematol 74: 173-178. 
Citation: Sharma RK, Puri V, Mutreja D, Kumar S, Chauhan P, et al. (2015) Applicability of a Single 5 Color Cytoplasmic Markers Tube as Primary Panel in Routine Immunophenotyping of Acute Leukemia. J Blood Disord Transfus 6: 309. doi:10.4172/2155-9864.1000309

Page 5 of 5

9. van der Schoot CE, von dem Borne AE, Tetteroo PA (1987) Characterization of myeloid leukemia by monoclonal antibodies, with an emphasis on antibodies against myeloperoxidase. Acta Haematol 78 Suppl 1: 32-40.

10. Dworzak MN, Fritsch G, Froschl G, Printz D, Gadner H (1998) Fourcolor flow cytometric investigation of terminal deoxynucleotidyl transferase-positive lymphoid precursors in pediatric bone marrow: CD79a expression precedes CD19 in early B-cell ontogeny. Blood 92: 3203-3209.

11. Mason DY, Cordell JL, Tse AG, van Dongen JJ, van Noesel CJ, et al. (1991) The IgM-associated protein mb-1 as a marker of normal and neoplastic B cells. J Immunol 147: 2474-2482.

12. Rani S, De Oliveira MS, Catovsky D (1988) Different expression of CD3 and CD22 in leukemic cells according to whether tested in suspension or fixed on slides. Hematol Pathol 2: 73-78.

13. Sartor M, Bradstock K (1994) Detection of intracellular lymphoid differentiation antigens by flow cytometry in acute lymphoblastic leukemia. Cytometry 18: 119-122.
14. Uckun FM, Ledbetter JA (1988) Immunobiologic differences between normal and leukemic human B-cell precursors. Proc Natl Acad Sci U S A 85: 8603-8607.

15. Galy A, Travis M, Cen D, Chen B (1995) Human T, B, natural killer, and dendritic cells arise from a common bone marrow progenitor cell subset. Immunity 3: 459-473.

16. Van Dongen JJM, Krissansen GW, Wolvers-Tettero IL, Comans-Bitter WM, Adriaansen HJ, et al. (1988) Cytoplasmic expression of the CD3 antigen as a diagnostic marker for immature T-cell malignancies. Blood 71: 603-612.

17. Drach D, Drach J, Glassl H, Gattringer C, Huber H (1993) Flow cytometric detection of cytoplasmic antigens in acute leukemias: implications for lineage assignment. Leuk Res 17: 455-461. 